$$
\begin{aligned}
& \text { 亮 }
\end{aligned}
$$

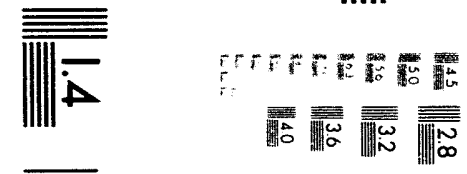

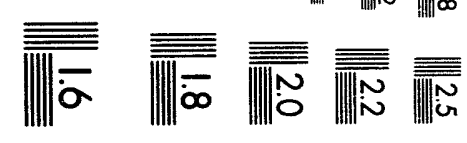



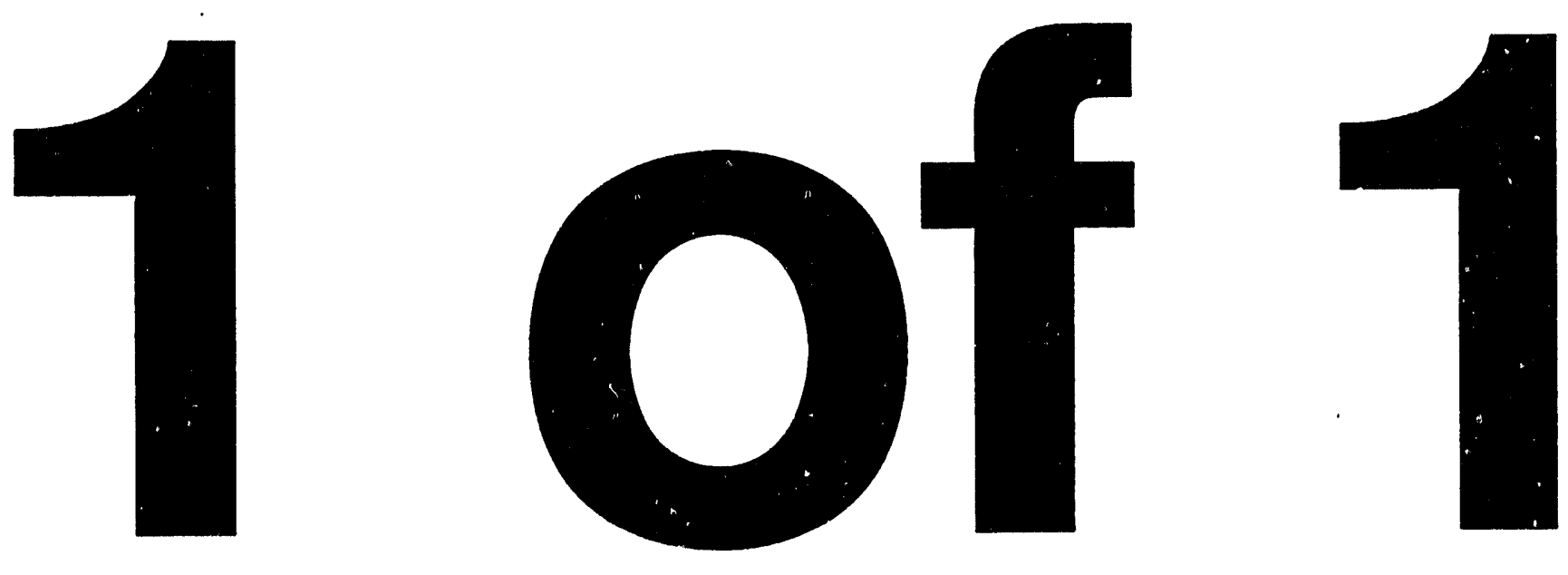


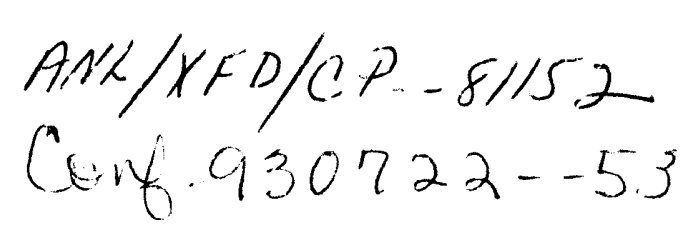

\title{
Comparison of technology for high-power laser mirrors and synchrotron radiation mirrors
}

\author{
H. E. Bennett \\ Physics Division, Rescarch Department \\ Naval Air Warfare Center Weapons Division \\ China Lake, California 93555-6001 \\ 1 \\ and \\ Ali M. Khounsary \\ Advanced Photon Source \\ Argonne National Laboratory \\ Argonne, Illinois 60439-4814
}

\begin{abstract}
There are obvious differences but some surprising similarities between high-power laser mirrors designed for use in the visible and infrared wavelengths and synchrotron mirrors designed for use at $x$-ray wavelengths. The use of synchrotron mirrors at grazing incidence results in a relaxation of figure, surface microroughness, and thermal heating tolerances relative to the wavelength of nearly two orders of magnitude. As a result, the tolerances become roughly comparable to those desired for high-power laser mirrors for the visible region of the spectrum. Experience gained over the years by laser mirror designers in substrate design, the influence of metallic coatings on thermal and optical performance and limiting values for surface microroughness may be helpful to designers of synchrotron systems, but exploitation of the promise of the new synchrotron systems still represents a challenging problem for researchers. The specifications and operational approaches to meeting them for the new Advanced Photon Source at Argonne National Laboratory are given.
\end{abstract}

\section{INTRODUCTION}

Mirrors used for high power laser applications and for synchrotron radiation systems have several things in common. First, they must withstand large amounts of radiation, some of which will be transformed into mirror heating. Second, they must have and maintain an excellent optical figure before and during operation so that the beam can be controlled. Third scattered energy from the mirrors must be minimized. Mirror cooling techniques are similar. To break up the stagnation layer at the coolant-faceplate interface direct impingement cooling is very effective and water is most commonly used as the cooling medium. Minimum thickness faceplates are used to reduce conductive resistance in the system.

There are also significant differences between laser and synchrotron mirrors. In an $x$-ray system the mirrors for the synchrotron system are typically used at grazing incidence. Laser mirrors are usually used either near normal incidence or at $45^{\circ}$. By going to grazing incidence synchrotron mirrors gain about two orders of magnitude in relaxation of tolerances relative to the wavelength both in optical figure requirements and in surface microroughness requirements. They also gain in reduction in intensity since the beam is spread out across the mirror and occupies about two orders of magnitude more area than it would at normal incidence.

The submitted manuscript has been authored by a contractor of the U.S. Government under contract No. W.31-109ENG-38. Accordingly, the U.S. Government retains a nonexclusive, royalty-free license to publish or reproduce the published form of this contribution, or allow others to do so, for U. S. Government purposes.
MASTER

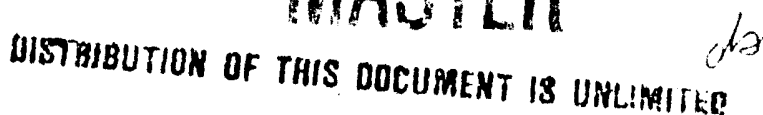


Although some laser mirrors are used with simple metallic coatings, the lowest absorption mirrors have a multilayer diclectric coating. With few exceptions $x$-ray synchrotron mirrors have metal surfaces. They are cither metallic or glass ceramics with a metallic coating. X-ray mirrors used in a grazing-incident configuration act as a low-pass spectral filter, that is, they reflect a large fraction of incident wide-spectium synchrotron $x$-ray beam below some cut-off photon energy. The low-bandpass property of grazing incident $x$ ray mirrors has received considerable attention recently because it allows such mirrors to be used as power filters for high power synchrotron beams. ${ }^{1-3}$

The cut-off energy for a grazing incidence $x$-ray mirror is a function of both the grazing incident angle and the reflecting surface electron density. This relationship comes directly from Maxwell's equations. At x-ray wavelengths matcrials have indices of refraction slightly less than unity. The real part of the complex dielectric constant is given by

$$
n^{2}-k^{2} \equiv n^{2}=1-\frac{4 \pi N c^{2}}{m \omega^{2}}
$$

where $N$ is the number of participating electrons per unit volume, $e$ the electron charge, $m$ its effective mass and $\omega$ the circular frequency of the light. Although the core electrons are too tightly bound to be affected by light at optical frequencies, all electrons for which the binding energy is less than $\frac{h \omega}{2 \pi}=h v$ contribute. Thus $N=\eta Z$, where $\eta$ is the number of atoms per unit volume and $\mathrm{Z}$ is the atomic number of the component material.

From Snell's law the critical angle $\phi$ (conventionally measured from the surface in $x$-ray work) is given for small angles by

$$
\phi=[2(1-n)]^{1 / 2}=\left(\frac{2 c}{\omega}\right)\left(\frac{\pi n Z}{m}\right)^{1 / 2}
$$

so that the larger the atomic number the larger the critical angle of the material. The critical angle is inversely related to the $x$-ray energy. Figure 1 depicts the computed reflectivity of a beryllium and a rhodium-coated mirror for grazing incident angles of 1.0 and $0.20^{\circ}$. Typically, coating with heavier metals or decreasing the grazing incident angle increases the cut-off energy. Grazing incidence mirrors with fixed incidence angle and different coatings or different grazing angles with a fixed coating can be used as low bandpass optical elements.

The broau spectrum $x$-ray beams generated by wigglers and undulators at third generation synchrotron radiation facilities contain kilowatts of power in very small solid angles. These thermal loads can produce unacceptable thermal distortion in the downstream optical components such as monochromators, mirrors, gratings, etc. Using a mirror at a grazing angle as the first optical element has at least four advantages. First, the grazing incident angle results in spreading the beam over a large area of the mirror, leading to a reduced incident heat flux and thus a less demanding cooling design for the mirror. Second, because it acts as a low pass filter, the thermal load on the downstream optical elements (that intercept the beam at larger angles) is substantially reduced. Third, the reflected beam, having a softer $x$-ray spectral range, requires less shiclding of the downstream beamline components ${ }^{3}$ and fourth the optical figure requirements are greatly reduced, as discussed later. 


\section{CataStrophic dama(iE THRESHOLd}

High power laser mirrors can be damaged catastrophically. X-ray mirrors do not fail in this way, They deteriorate over time through chemical reactions at the surface, thermally induced cracks, degradation of coatings, etc. For laser mirrors considerable work has gone into catastrophic damagc. Usually damage occurs at imperfections in the mirror surface. From a practical point of view the occurrence of microscopic damage is not so important as the spread of such damage in a catastrophic manner. For continuous wave operation (or pulsed operation when the pulse rate is high enough so that an average heat rate can be assumed) the growth of defects can be predicted from simple thermal analysis. ${ }^{4}$ The principle is illustrated in Fig. 2. If poor thermal contact exists between the defect and its surroundings, it will heat rapidly and may melt or fracture the surface. If good thermal contact is established by this process, the growth of the defect may be contained at this point ard no further growth will occur. The limiting flux value depends on the type of defect and the substrate on which is is found. Some calculated values for ULE glass substrates, which have very low conductivity, and copper substrates, which have a high conductivity, are given in Fig. 3. These values appear to be in reasonable agreement with experiment. A similar expression, corrected for angle of incidence, might apply to synchrotron mirrors. However, long before survival of the synchrotron mirror becomes an issue the acceptable thermal distortion, or more accurately the slope error, determines the limiting flux the mirror can handle.

\section{THERMAL HEAT LOADING}

Both high-reflectance metallic and multilayer-coated mirrors are used in high-power laser mirrors. Synchrotron mirrors are usually metals or metal coated. At near-normal incidence metallic reflection is much lower in the $x$-ray region than at visible or infrared wavelengths, but at grazing angles it is typically close to unity. For example, for $\mathrm{Be}$ and $\mathrm{Rh}$ mirrors the reflectance is typically greater than 0.9999. Multilayer-coated dielectric mirrors are beginning to be used at $x$-ray wavelengths for some applications. For laser mirrors the lowest absorptances are obtained by using multilayer dielectric coatings. Multilayer reflector designs are usually determined on a computer using impedance theory and can be quite complicated. A basic high reflector coating design employs alternating high- and lowindex transparent films with optical thicknesses of a quarter of a wavelength. The high or low index film next to the metal surface, if there is a metal surface, is modified in thickness to compensate for the phase change of the metal. The standing wave electric fields in a multilayer film of this type are shown in Fig. 4. Reflectances differing from unity by a few parts in $10^{4}$ can be achieved with such multilayers. The mathematics necessary for the multilayer calculations, which are iterative, ucually obscures the physics of the situation. However, for quarter wavelength thick coatings a closed form, relatively simple expression has been worked out. For a dielectric substrate with the high-index film next to it the normal incidence reflectance $R$ is 5

$$
\begin{aligned}
R= & -\left[\frac{\lambda}{2}\left(\beta_{L}+\beta_{H}\right)+4 A_{H L}\right]\left[1-\left(\frac{n_{L}}{n_{H}}\right)^{2 N+1}\right] \frac{1}{n_{H}^{2}-n_{L}^{2}} \\
& -4 \frac{n_{S}}{n_{H}^{2}}\left(\frac{n_{L}}{n_{H}}\right)^{2 N}\left(1+A_{A s}\right)-\left(\frac{4 \pi \sigma}{\lambda}\right)^{2}
\end{aligned}
$$


where $\lambda$ is the wavelength, $\beta_{L}$ and $\beta_{H}$ the absorption coefficients of the low and high index layers of index $n_{L}$ and $n_{H}$, dielectric substrate index $n_{S}$ and $A_{H L}$ and $A_{H S}$ the interface absorption between high and low index layers and the high index layer and the substrate respectively. The $\mathrm{mm}$ surface roughness of the substrate, assumed to be replicated in each of the layers, is $\sigma$. A similar expression can be derived when the low index film is next to the substratc. For a metal or metal-coated substrate $\mathrm{n}_{S}$ is replaced by the (real) metal/high index layer impedance. In looking at these expressions the physics of the situation becomes clear. As the number of layer pairs $\mathrm{N}$ becomes large, the absorption of the multilayer is determined by the absorption coefficients of the individual layers and by the interface absorption $A_{H L}$. The reflectance is determined by the absorptance and the scattering, which is a function of the rms roughness of the substrate (plus additional imperfections in the films, neglected here). It will approach a constant value independent of the number of film pairs and can only be increased by improving the quality of the deposited films and the smoothness of the substrate. In the visible or infrared regions of the spectrum a good multilayer film will have one or more orders of magnitude less absorption than any uncoated metal film, which means that unless the dielectric is defect-limited, a multilayer coated mirror can withstand over an order of magnitude higher incident heat flux than can a metal coated laser mirror. For this reason the best high power laser mirrors are multilayer coated. Damage thresholds depend on materials, wavelength, preconditioning, $\mathrm{cw}$ or pulse and pulse length, layer absorption, defects and other parameters. A typical value of the damage threshold for good laser mirrors is $10 \mathrm{Kw} / \mathrm{cm}^{2}$ for continuous wave mirrors and $10 \mathrm{~J} / \mathrm{cm}^{2}$ for mirrors exposed to picosec pulses. Order of magnitude variations both up and down are observed in both cases.

\section{MIRROR DISTORTION PER UNIT OF ABSORBED FLUX}

No matter how effective the coating is in reflecting incident heat flux, some of it will be absorbed. It is thus important to understand the thermal distortion of the mirror which will occur per unit of heat flux absorbed. This thermal distortion is a strong function not only of material properties of the mirror and faceplate thickness but of the coolant used and the heat exchanger design. The thermal distortion coefficient is defined as the ratio of the laserinduced surface normal distortion to the absorbed heat flux. It is often expressed in $\mathrm{A} / \mathrm{watt} / \mathrm{cm}^{2}$. A good cooled mirror will have a distortion coefficient of $10 \mathrm{~A} / \mathrm{w} / \mathrm{cm}^{2}$ or less, and considerably lower values have been reported. The first consideration is the mirror material. A convenient figure of merit for mirror materials is the ratio of the thermal expansion coefficient to the thermal conductivity. Values for some useful materials are given in Table $1.6,7$

On this basis the most promising substrate materials are single crystal silicon and reaction sintered silicon carbide. The way in which the material is formed is very important, as evidenced by the $\mathrm{Si}$ data. CVD silicon ${ }^{6}$ is less than half as good as single crystal silicon. Again and again the importance of materials preparation on material properties is evident in optics.

\section{DISCLAIMER}

This report was prepared as an account of work sponsored by an agency of the United States This report was prepared as an account of work sponsored by an agency of the United States employees, makes any warranty, express or implied, or assumes any legal liability or responsiemploses, or process disclosed, or represents that its use would not infringe privately owned rights. Referprocess disclosed, or represents that its use would not infricese or service by trade name, trademark manufacturer, or otherwise does not necessarily constitute or imply its endorsement, recommanufacturer, or otherwise does not necessarily constitutes or any agency thereof. The views and opinions of authors expressed herein do not necessarily state or reflect those of the United States Government or any agency thereof. 
TABLE 1. PROPERTIES OF CANDIDATE MIRROR MATERIALS

\begin{tabular}{l|c|c|c}
\hline Material & $\begin{array}{c}\text { Thermal Expansion } \\
\text { Cocfficicnt } \\
\alpha\left(10^{-6} /{ }^{\circ} \mathrm{C}\right)\end{array}$ & $\begin{array}{c}\text { Thermal } \\
\text { Conductivity } \\
\mathrm{k}\left(\mathrm{W} / \mathrm{cm}{ }^{\circ} \mathrm{C}\right)\end{array}$ & $\begin{array}{c}\text { Figure } \\
\text { of Merit } \\
\alpha / \mathrm{k}\end{array}$ \\
\hline Single crystal silicon & 2.10 & 1.53 & 1.37 \\
\hline Reaction sintered SiC & 2.45 & 1.50 & 1.63 \\
\hline Tungsten & 4.5 & 1.69 & 2.66 \\
\hline Zerodur & 0.05 & 0.0164 & 3.05 \\
\hline $\begin{array}{l}\text { CVD finc grain } \\
\text { polycryst Si }\end{array}$ & 2.67 & 0.84 & 3.18 \\
\hline Molybdenum & 4.86 & 1.34 & 3.35 \\
\hline Copper & 16.7 & 4.00 & 4.17 \\
\hline Beryllium & $11 / 2$ & 2.10 & 5.33 \\
\hline Fused quartz & 0.41 & 0.014 & 29.3 \\
\hline
\end{tabular}

Of the materials listed fused silica has the worst figure of merit, which is a surprise. It is important to remember, however, that this figure of merit is for distortion, not damage threshold. For example, fused silica has very low thermal conductivity and coatings on this substrate may get quite hot. However, coatings on fused silica may have a coating damage threshold which is only 4 times lower than for silicon even though its distortion figure of merit is over 20 times worse than silicon. Therefore, $\alpha / k$ should not be used as a damage threshold predictor. As a distortion indicator $\alpha / k$ is a valid figure of merit. For example, interferometric measurements have verified ${ }^{8}$ that cooled coated silicon and silicon carbide mirrors have about half the thermal distortion of similar cooled coated molybdenum mirrors at the same flux level. The ratio of their figures of merit is 0.41 and 0.49 , respectively.

The design of the cooling flow geometries is of great importance in determining the thermal distortion coefficient of a cooled mirror. Faceplate thickness is also critical. Thicknesses of $0.6 \mathrm{~mm}$ are feasible for some materials, for example, although $1.0 \mathrm{~mm}$ thicknesses are more common. Closely spaced coolant channels under the faceplate are a common coolant design. Channels may be perhaps a $\mathrm{mm}$ wide, $1 / 3 \mathrm{rd} \mathrm{mm}$ in height separated by a $1 / 3 \mathrm{rd} \mathrm{mm}$ wall. Microchannels, which are mostly used in the electronics industry for VSLI applications, may be only $50 \mu \mathrm{m}$ wide and can handle $23 \mathrm{w} / \mathrm{mm}^{2}$, with a temperature rise of perhaps $100^{\circ} \mathrm{C}$ between faccplate and backing plate (about $0.2 \mathrm{w} / \mathrm{mm}^{2} /{ }^{\circ} \mathrm{C}$ in $\mathrm{x}$-ray nomenclature). Faceplate thicknesses in this case are about $0.1 \mathrm{~mm}$, too thin for optical polishing. A problem with channel designs, particularly if the channels are of larger dimensions, is the stagnation layer of coolant next to the faceplate wall. This stagnation layer can be broken up by offsetting the channels periodically so the flow is very turbulent (the so-called plate-fin design) or by making a high density of small posts through which the coolant must pass. The so-called "impingement cooling" where the coolant flows up to strike the faceplate and 
disrupt this stagnation layer, is very effective. Cooled mirror designs continue to evolve, ${ }^{9}$ and at a given distortion level reductions of an order of magnitude in flow rate (with resultant decrease in jitter) has been reported. 10

Other parameters are the coolant flow rate, the number of layers of channels, and the type of coolant used. Cooling increases nearly lincarly with flow rate, and rates of hundreds of gallons per minute are sometimes used for large mirrors. However the jitter of the optics also increases with flow rate, so large flow rates cannot always be tolerated. Two or even three layers of channels are often used. Again jitter is a problem. Water, with appropriate additives to reduce corrosion, is the favorite cooling medium. It has the largest heat capacity of any common fluid and is also inexpensive. Some work has been done with coolants which undergo a phase change near room temperature, 11,12 utilizing the associated latent heat for cooling. Jitter can be reduced significantly using this approach.

\section{THERMAL DISTORTION FOR AN OPTICAL TRAIN}

In imaging optics tolerances are usually determined using the Rayleigh quarter-wave criterion. However for power optics, where the objective is to transmit the maximum amount of power to a focussed spot, the Strehl relation is more appropriate. It may be stated 13

$$
\frac{I}{I_{0}}=1-\left[\frac{2 \pi}{\lambda}\right]^{2} \rho^{2}
$$

where $I=$ the peak intensity in the focussed spot, $I_{0}=$ the theoretical peak intensity if all optical components were perfect and $\rho$ is the rms wave front error introduced by the optical train. Assume that polishing errors in the optical figures of each of the $N$ components are of the same magnitude but occur randomly over the surface and are thus uncorrelated between different components. The thermally induced figure errors between components result from the same beam and thus are assumed to be correlated. Then

$$
\rho^{2}=N\left(\rho_{\zeta}\right)^{2}+\left(N \rho_{t}\right)^{2}
$$

$\rho_{\zeta}$ is the polishing induced wave front error in each component and $\rho_{t}$ is the thermally induced wave front error. Since for a mirror the wave front error is twice the figure error and the peak to valley figure errors are approximately $2 \sqrt{2}$ times the rms figure errors, the allowable peak to valley figure error $\zeta$ for a mirror is approximately

$$
\zeta=\frac{\lambda^{2}}{10 \pi^{2} N}-2 N \rho_{t}^{2}
$$

Using the thin shell model for thermal distortion, the limiting single pulse energy density $I_{q}$ for mirrors for times $t$ short compared to the thermal time constant of the faceplate of the cooled substrate is then 14

$$
I_{q} t=\frac{\rho C_{p}\left[\frac{\lambda^{2}}{5 \pi^{2} N}-2 \tau f^{2}\right]^{1 / 2}}{2(1-R) \alpha\left[2 N\left(1+3 \chi+3 \chi^{2}\right)\right]^{1 / 2}}
$$


Where $\tau_{f}$ is the average peak to peak figuring error per mirror, defined as $2 \sqrt{2}$ times the rms figure error of the mirror, and $\chi=\left(1 / \sqrt{\pi}(b / 1)^{2}\right.$ is the mirror radius, where $b$ is the $1 / c^{2}$ radius of the Gaussian beam striking the mirror. The limiting peak encrgy density for unacceptable distortion is then directly proportional to the specific heat $C_{p}$ and density $\rho$ of the mirror substrate and inverscly proportional to its absorption $(1-R)$ and expansion coefficient $\alpha$. If the initial figure crror $\tau_{f}$ is negligible, it is also directly proportional to the wavelength and inversely proportional to the square root of the number of components. If the component is used at an angle of $45^{\circ}$ and focal shift can be tolerated, the critcria on surface figure are relaxed 8 by a factor of 3.3 and at $60^{\circ}$ by a factor of 3.4 , as illustrated in Fig. 5. If the component is used at grazing incidence the wave front error caused either by random irregularities or spherical power approaches zero. For example, at an angle of $1^{\circ}$ grazing the multiplicative factor is $\cos 89^{\circ}=0.017$ and at $0.2^{\circ}$ it is 0.003 . For the same figure error the wavelength at grazing incidence can then be approximately two orders of magnitude shorter than at normal incidence. Reducing a visible wavelength by three orders of magnitude puts it in the $x$-ray region, 0.3-10 A where the Advanced Photon Source (APS) operates.

Figure 6 illustrates 15 the calculated peak pulsed energy density (from Eq. 7), which a molybdenum mirror could withstand without exceeding the distortion limits of a ten element optical train as a function of infrared wavelength. The calculation is approximate. It neglects heat conduction into the mirror, assumes that an equal error budget is allotted to each component, and assumes that laser damage to the component is not a factor. The initial figure error at visible wavelengths is assumed to be $\lambda / 4, \lambda / 8$ and 0 for the uncoated molybdenum mirror. The 0 figure error for the uncoated mirror data is compared to that expected for a multilayer coated mirror with high absorption, $0.2 \%$. Multilayer mirrors with much lower absorption can be produced.

There are significant differences between the threshold values for peak energy densities for uncoated metal laser mirrors also, as illustrated in Fig. 7. For short pulse lengths the damage threshold of the material may be exceeded. For longer pulse lengths it is the allowed thermally induced distortion, which limits the pulsed energy density which the mirror can withstand. For even longer times it will be limited by the heat transfer ability of the cooling system. The damage threshold in these theoretical curves depends not only on melting temperature but on conductivity and other factors, as illustrated by copper, which has a higher theoretical damage threshold than molybdenum even though it has a much lower melting temperature. Both have about the same threshold for thermal distortion as illustrated by the horizontal curves. The danger in relying on calculated values for damage thresholds is illustrated in Fig. 8. Depending on surface condition the experimental values 16 for the thresholds vary here by almost a factor of 4 .

The absorption as well as the damage threshold of metal surfaces may vary significantly depending on surface condition. For example, at wavelengths longer than $3 \mu \mathrm{m}$ in the infrared the absorption of silver or gold may decrease as much as one third for super smooth surfaces (a few $\AA \mathrm{rms}$ ) as a result of the anomalous skin effect.17 In the blue and near ultraviolet the same effect, excitation of surface plasmons, can increase absorption by as much ${ }^{18}$ as a factor of 10 . Even simple crystalline distortion caused by surface finishing can reduce the mean free path of the conduction electrons, alter the Fermi surface and cause an increase in absorption. An example of surface distortion when preparing a copper surface by diamond turning, a low distortion technique for preparing optical surfaces, is seen in Fig. 9. At $x$-ray wavelengths the absorption tied to the core energy levels in individual atoms will not be affected by the cooperative electronic effects which affect the optical properties of 
the material at longer wavelengths. However at grazing incidence the optical properties are intimately connected with the crystal structure of the matcrial and at grazing incidence the resulting absorption should be closely tied to sample condition. Attention to the conditions of sample preparation is thus essential for both laser and $\mathrm{x}$-ray mirrors.

\section{THERMAL LOAD ON HIGH HEAT LOAD X-RAY MIRRORS}

Unlike laser mirrors, which reflect but a small fraction of the incident beam, $\mathrm{x}$-ray mirrors can absorb a substantial portion (and in the case of multilayer mirrors almost all) ${ }^{19}$ of the incident $x$-ray beam.

The angular power distribution (in $\mathrm{kW} / \mathrm{mrad}^{2}$ ) of an $\mathrm{x}$-ray beam generated by a wiggler or an undulator is given by 20

$$
\frac{d^{2} P}{d \theta d \psi}=10.84 B_{0}[T] E_{r}^{4}[G e V] I[A] N G(K) f_{K}(\theta, \psi)
$$

where $B_{0}$ is the peak magnetic field (in Tesla), $E_{r}$ is the storage :ing energy, $I$ is the electron (or positron) current in the storage ring, and $\mathrm{N}$ is the number of undulator or wiggler periods. $K$ is the so-called deflection parameter, given by

$$
\mathrm{K}=0.934 \lambda_{\mathrm{u}}[\mathrm{cm}] \mathrm{B}_{0}[\mathrm{~T}]
$$

where $l_{u}$ is the undulator or wiggler period length. The function $G(k)$ in Eq. 8 has a value of zero at $\mathrm{K}=0$ and asymptotically approaches unity for $\mathrm{K}>1.0$ It is given by 20

$$
G(K)=\frac{K^{7}+24 K^{5 / 7}+4 K^{3}+16 K / 7}{\left(1+K^{2}\right)^{7 / 2}}
$$

The function $\mathrm{f}_{\mathrm{k}}(\theta, \psi)$ in Eq. 8, which describes the angular behavior of the power profile is a complex integral equation. It is normalized such that $f_{K}(0,0)=1$.

The total power (in $\mathrm{kW}$ ) of an $\mathrm{x}$-ray beam from a wiggler or an undulator is given by

$$
\mathrm{P}_{\mathrm{T}}=0.633 \mathrm{E}_{\mathrm{r}}^{2}[\mathrm{GEV}] \mathrm{B}_{0}^{2}[\mathrm{~T}] \mathrm{L}[\mathrm{m}] \mathrm{I}[\mathrm{A}] \text {, }
$$

where $\mathrm{L}$ is the device length.

In Table 2, the device parameters for an undulator designed for installation at the APS are given.21 The angular power profile of this beam is shown in Fig. 10. The peak power density is about $134 \mathrm{~kW} / \mathrm{mrad}^{2}$, and the total power is $3.8 \mathrm{~kW}$. As seen from Fig. 10, the beam from this undulator is confined to a very narrow solid angle. The on-axis full width at half maximum (FWHM) of this beam is about $95 \mu \mathrm{rad}$ vertically and $270 \mu \mathrm{rad}$ horizontally. 
Table 2. Parameters for the Undulator $A$ at the APS 1

\begin{tabular}{l|c}
\hline Devicc & Undulator A \\
\hline Undulator Period, $\lambda_{\mathrm{u}}[\mathrm{cm}]$ & 3.3 \\
\hline Number of Periods, $\mathrm{N}$ & 72 \\
\hline Device Length, L $[\mathrm{m}]$ & 2.4 \\
\hline Minimum Gap $[\mathrm{cm}]$ & 1.15 \\
\hline Deflection Parameter, K & 2.23 \\
\hline Maximum Field, $\mathrm{B}_{0}[\mathrm{~T}]$ & 0.72 \\
\hline First Harmonic Energy $\mathrm{E}_{1}[\mathrm{keV}]$ & 4.2 \\
\hline Characteristic Energy, $\mathrm{E}_{\mathrm{C}}[\mathrm{keV}]$ & 23.5 \\
\hline Total Beam Power $[\mathrm{kW}]$ & 3.8 \\
\hline Peak Power Density $\left[\mathrm{kW} / \mathrm{mrad}^{2}\right]$ & 134 \\
\hline $\begin{array}{l}\text { Peak Heat Flux (on a } 1.25^{\circ} \mathrm{grazing} \text { incidence } \\
\text { mirror placed } 30 \mathrm{~m} \text { from the source) }\left[\mathrm{W} / \mathrm{mm}^{2}\right]\end{array}$ & 3.2 \\
\hline
\end{tabular}

The normal incident heat flux on a mirror placed $30 \mathrm{~m}$ from the source is $150 \mathrm{~W} / \mathrm{mm}^{2}$. At a grazing incident angle of $1.25^{\circ}$, this translates to $3.2 \mathrm{~W} / \mathrm{mm}^{2}$. This high heat flux, most of which may be absorbed by the mirror, can lead to severe thermal distortions. Thus, design of mirrors with excellent cooling capabilities to handle this heat load while providing acceptable performance is a major engineering challenge in synchrotron optics. $\mathrm{Cw}$ laser optics can handle fluxes of $100 \mathrm{w} / \mathrm{mm}^{2}$ without damaging but these values drop drastically for pulsed radiation, reaching typical values of $0.1 \mathrm{w} / \mathrm{mm}^{2}$ for picosec pulses. $\mathrm{Cw}$ distortion levels are lower than damage levels, as discussed previously and illustrated in Fig. 7.

\section{OPTICAL TOLERANCES FOR X-RAY MIRRORS}

The thermal distortions on a high heat load $x$-ray mirror must be controlled to prevent distortions of the $x$-ray wave front beyond acceptable limits. These limits are determined by the configuration of beamline optics, as well as the $x$-ray source characteristics. Consider an $x$-ray source whose vertical and horizontal dimensions are represented, respectively, by $2 \delta_{v}$ and $2 \delta_{\mathrm{h}}$, where $\delta_{\mathrm{v}}$ and $\delta_{\mathrm{h}}$ are the source size vertical and horizontal standard aeviations. The maximum allowable slope errors (over long surface wavelengths) in an otherwise optically perfect element can be estimated by requiring a virtual image of the source in a plane deflection mirror that is not significantly larger than the actual source. This leads to the following relationships for sagittal, $\Delta s$, and tangential, $\Delta t$, slope errors:

$$
\begin{aligned}
& \Delta_{\mathrm{S}}<\frac{2 \sigma_{\mathrm{V}}}{3 \mathrm{r} \theta} \\
& \Delta_{\mathrm{t}}<\frac{2 \theta_{\mathrm{h}}}{2 \mathrm{r}},
\end{aligned}
$$


where $r$ is the source 10 mirror distance, and $\Theta$ (in radians) is the angle of incidence.

In practice, these slope errors should be between a third and a tenth of the quantitics on the right hand side of Eq. 12 to avoid appreciable slope-crror-induced image broadening.

For the APS beams, the source dimensions are $\delta_{\mathrm{v}}=91 \mu \mathrm{m}$ and $\delta_{\mathrm{h}}=342 \mu \mathrm{m}$. Taking a value of $\mathrm{r}=$ $30 \mathrm{~m}$ and $\Theta=1.25^{\circ}$ (22 $\left.\mathrm{mrad}\right)$, then

$$
\begin{aligned}
& \Delta_{\mathrm{S}} \approx 0.1\left(\frac{2 \sigma_{\mathrm{v}}}{2 \mathrm{r} \theta}\right)=14 \mu \mathrm{rad} \\
& \Delta_{\mathrm{l}} \approx 0.1\left(\frac{2 \sigma_{\mathrm{h}}}{2 \mathrm{r}}\right)=1.1 \mu \mathrm{rad} .
\end{aligned}
$$

The much larger acceptable slope error in the sagittal directions is due to the so-called "forgiveness factor," which is exploited in synchrotron beamline design by using horizontal deflecting mirrors so that the large thermally induced slope error is in the sagittal plane.

It should be noted that, in practice, these slope error budgets include both the fabricationrelated errors as well as the thermally induced errors. Since the minimum fabrication rms slope errors for an average sized mirror are currently about $1 \mu \mathrm{rad}$, little room is left for thermally induced errors, and this clearly illustrates the need for cleverly engineered cooled $x$-ray mirrors.

\section{SCATTERED LIGHT}

The total integrated scatter from an optically polished surface is well represented in the visible region of the spectrum by scalar scattering theory 22,23

$$
\text { TIS }=\left[\frac{4 \pi \delta(\cos \theta)}{\lambda}\right]^{2}
$$

where $\theta$ is measured from the normal to the surface. In $x$-ray work the angle is measured from the surface so that $\left(90^{\circ}-\psi\right)$ should be substituted for $\theta$. Expanding gives $\cos \theta=\cos$ $90^{\circ} \cos \psi-\sin 90^{\circ} \sin \psi=\sin \psi=\psi$. At grazing incidence the scattered light is then given by

$$
\text { TIS }=\psi^{2}\left[\frac{4 \pi \delta}{\lambda}\right]^{2}
$$

A plot of the wavelength dependence of 40 and $10 \AA \mathrm{rms}$ surfaces for TIS values of 1 and $10 \%$ is seen in Fig. 11. As has been pointed out previously, 24 in the soft $x$-ray region at angles of incidence of $1^{\circ}$ or less the scattering levels are similar to those of interest in the visible region. Furthermore the spatial wavelengths of the surface features are similar, as seen from Fig. 12. The surface spatial wavelengths give rise to light scattered more and more closely about the specular direction as we decrease the wavelength. For example, in the visible at $5000 \AA$ wavelength light from surface spatial wavelengths of $28 \mu \mathrm{m}$ is scattered into a cone angle of $1^{\circ}$ about the specular direction. At a soft $x$-ray wavelength of $10 \AA$ it is scattered into a cone with half angle $7 \mathrm{sec}$ of arc about the specular direction. At the other extreme, light scattered at $90^{\circ}$ to the specular direction at visible wavelengths is scattered into a cone with half angle 7 minutes of arc. These results are of great importance to $x$-ray optics since they show that the range of spatial wavelengths and roughness values which 
are important in the visible region of the spectrum and upon which a great deal of work has been done are the ones that are important in the soft $x$-lay region of the spectrum for grazing incidence systems. Surface spatial wavelengths shorter than those accessible to visible wavelengths may also be important at $x$-raly wavelengths, and cannot be measured using optical techniques. Despite this uncertainty, an optical surface measurement is useful in cvaluating surfaces used at $x$-ray wavelengths.

\section{PRACTICAL EXPERIENCE IN BEAMLINE DESIGN}

Microroughness is commonly specified for $x$-ray mirrors. Values typically vary from 0.5 to $10 \AA$ rms. Slope errors are also specified. However, it is not possible to accurately predict the performance of a grazing incidence $x$-ray mirror surface defined only by the optically measured values of this pair of parameters.

Extensive work by Takacs and co-workers 25,26 in recent years, not only theoretical but also in developing metrology techniques and instrumentation, has led to a much better understanding of the relationsliips between the performance of grazing-incidence $x$-ray mirrors and their measured topological properties. The latter, expressed in terms of power spectral density (PSD) function over spatial wavelengths of a few micrometers to a meter or so, are particularly useful in improving the fabrication techniques to produce the high tolerance optics that the advanced $x$-ray beamlines demand.

In addition to these problems, the addition of thermal loading and particularly the high heat fluxes of wiggler and undulator beams make the task of designing high performance grazing-incidence cooled $x$-ray mirrors challenging, even if the surface fabrication and characterization issues are fully resolved.

Presently, the design of a high heat load mirror as the first optical element on an APS undulator beamline is underway. This mirror is exposed to an undulator beam with a total incident power (after an aperture) of about $2 \mathrm{~kW}$ and a peak incident heat flux of $3.2 \mathrm{~W} / \mathrm{mm}^{2}$. A total slope error of no more than $2 \mu$ rad (including thermally induced) with an rms roughness of $6 \AA$ (over $5-5000 \mu \mathrm{m}$ spacial wavelengths) is specified. The design of this mirror is outlined elsewhere in these proceedings, and it is expected that the mirror would meet the said specifications. The characterization of this mirror under high heat load is being planned. The excellent Thermal Distortion Test Facility at Kirtland Air Force Base will be used to accurately simulate the thermal load on the mirror and obtain both temperature and distortion information for comparison with computational results.

\section{CONCLUSIONS}

There are obvious differences but some surprising similarities between high power laser mirrors designed for use in the visible and infrared wavelengths and synchrotron mirrors designed for use at $x$-ray wavelengths. The use of synchrotron mirrors at grazing incidence results in a relaxation of normal incidence figure, surface microroughness and thermal heating tolerances relative to the wavelength of nearly two orders of magnitude, partially compensating for the disparity of $\mathrm{x}$-ray and visible wavelengths. As a result, the tolerances on $\mathrm{x}$-ray mirrors become comparable within an order of magnitude or so to those required for laser mirror designed for the visible region. Experience gained over the years by laser mirror manufacturers in substrate design and fabrication, effects of coating procedures on thermal and optical performance and reduction of surface microroughness may be helpful to designers of synchrotron systems, but exploitation of the promise of the new synchrotron systems still represents a challenging problem for researchers. 


\section{REFERENCES}

1. R. DiGennero, B. Gec, J. Guigli, H. Hogrefe, and M. Howells, Nucl. Instrum. and Meth. A266, 498-506 (1988).

2. S. L. Hulbert and S. Sharma, Opt. Eng. 27, $433-439$ (1988).

3. W. Yun, A. Khounsary, B. Lai, and E. Gluskin, "Use of a mirror as the first optical component of an undulator beamline at the APS," Argonne National Laboratory Report ANL/APS/TB-2, 1992.

4. H. E. Bennett, "Insensitivity of the Catastrophic Damage Threshold of Laser Optics to Dust and Other Surface Defects," in Proceedings of the Laser Induced Damage in Optical Materials, 1980. Pp. 256-264.

5. H. E. Bennetl and D. K. Burge, "Simple expressions for predicting the effect of volume and interface absorption and of scattering in high reflectance or antireflectance multilayer coatings," J. Opt. Soc. Am 70, 268 (1980).

6. K. C. Sun, R. L. Wahl, F.M. Anthony and T.W. Tonnessen, "Silicon Mirrors for Advanced Free-Electron Lasers," in Proceedings of the Topical Meeting on High Power Laser Optical Components, 30-31 October 1989, NWC TP 7080, December 1990, pp. 124-138.

7. American Institute of Physics Handbook. D. E. Gray, ed., pp. 4-119 to 4-140 and 4-154 to 4-158, McGraw-Hill, New York (1972).

8. J. R. Bolch, "Comparison of Molybdenum and Silicon Carbide Cooled Laser Mirrors Based on Thermal Distortion Testing." in Proceeding of the High Power Optical Components Meeting (Oct 1984). AFWAL-TR-85-4141, pp. 272-282, July 1985.

9. M. A. Ealey and J. A. Wellman, "Isoflow Mirror Tests Conducted at the Thermal Distortion Test Flow Facility," in Proceedings of the Topical Meeting on High Power Laser Optical Components, 22-23 October 1990, J. L. Stanford, ed., NWC TP 7190, September 1991, p. 82.

10. G. P. Eiler and P. B. Wilkinson, "Advanced Heat Exchanger Materials and Design for Superior Thermal Performance with Ultralow Flowrate and Jitter," op. cit., p. 83.

11. J. H. Rosenfeld, M. E. Meadows and P. C. LaDelfe, "Advances in Pumped Phase Change Mirror Cooling," op. cit., pp. 66-77.

12. J. H. Rosenfeld, M. E. Meadows, J. L. Stapp and P. C. LaDelfe, Distortion Tests of a Pumped Two-phase Cooled Mirror," in Proceedings of the Topical Meeting on High Power Optical Components, 21-22 October 1991, D. L. Decker, ed., NWC TP 8025, October 1992, p. 156.

13. M. Bom and E. Wolf, Principles of Optics, 1st ed., p. 468. Pergamon Press, New York (1959).

14. H. E. Bennett, "Optical Figure Requirements for Laser Mirrors Used at Oblique Incidence," in Proceedings of the Laser Induced Damage in Optical Materials 1982, H. E. Bennell, A. Guenther, D. Milam, and B. Newnam, eds., NBS Special Publication 669, 228-233.

15. H. E. Bennet,. "Thermal Distortion Thresholds for Optical Trains Handling High Pulse Powers," in Proceedings of the Laser Induced Damage in Optical Materials 1976. A. J. Glass and A. Guenther, eds., NBS Special Publication 462, 11-24. 
16. M. J. Soilcau and V. Wang. "Improved Damage Thresholds for Metal Mirrors," Appl. Opt. 13, 1286 (1974).

17. H. E. Bennett, J. M. Bennett, E. J. Ashley, and R. J. Motyka, "Verification of the Anomalous Skin Effect Theory for Silver in the Infrared," Phys. Rev. 165, 755 (1968).

18. J. L. Stanford, "Determination of Surface-Film Thickncss from Shift of Optically Excited Surface Plasmon Resonance," J. Opt. Soc. Am. 60, 49-53.

19. J. B. Kortright, "Mirrors as Power Filters," Proc. SPIE 1345, 38-41 (1990).

20. K.-J. Kim, Nucl. Instrum. and Meth. A246, 67-70 (1986).

21. B. Lai, A. Khounsary, R. Savoy, L. Moog, and E. Gluskin, "Undulator A Characteristics and Specifications," Argonne National Laboratory Report ANL/APS/TB-3, 1993.

22. H. Davies, Proc. Inst. Elec. Engrs. (London) 101, 209 (1954).

23. H. E. Bennett and J. O. Porteus, "Relation Between Surface Roughness and Specular Reflectance at Normal Incidence," J. Opt. Soc. Am 51, 123 (1961).

24. H. E. Bennett, "Proposed Use of Visible Optical Techniques to Evaluate the Surface Finish of X-Ray Optics," Opt. Engr. 19, 610-615 (1980).

25. P. Z., Takacs, Nucl. Instrum. and Meth. A246, 227-241 (1986).

26. P. A. Takacs and E. L. Church, Nucl. Instrum. and Meth. A291, 253-264 (1990).

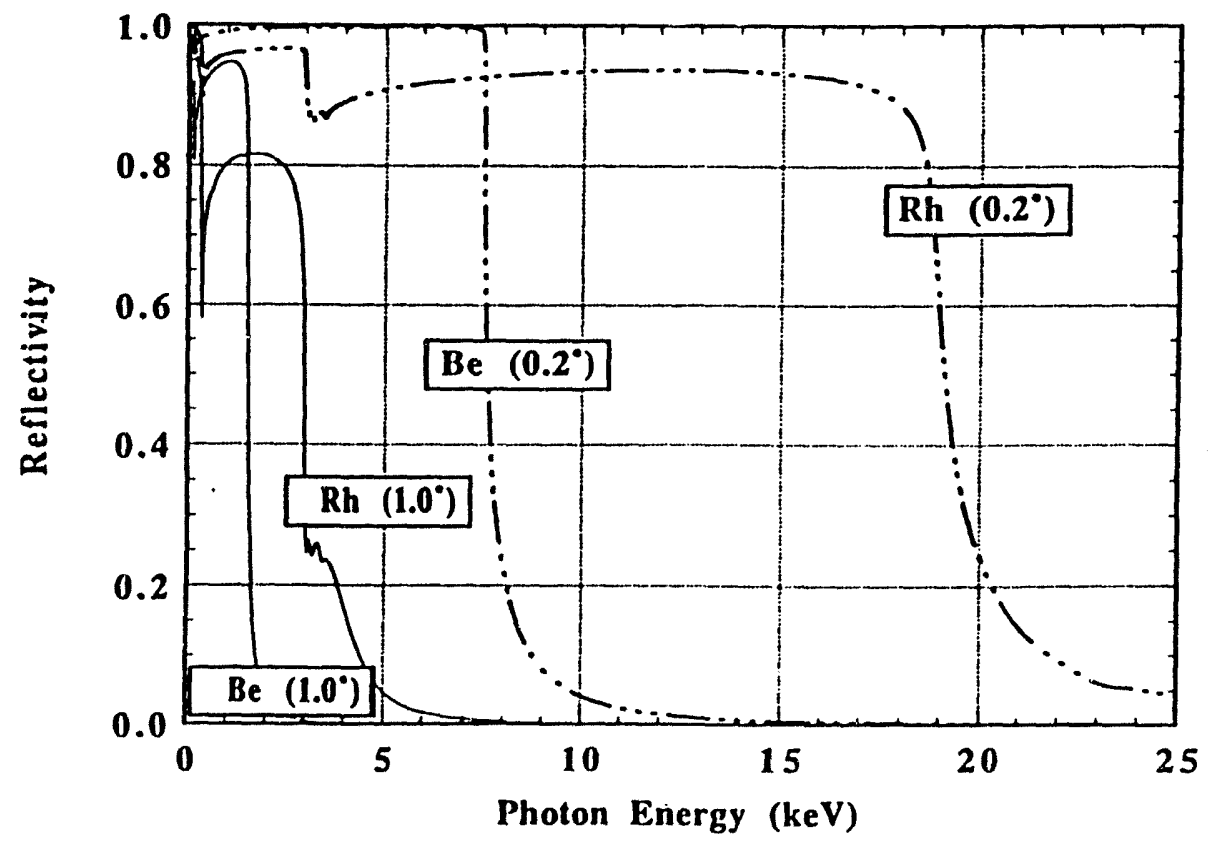

Figure 1. Computed reflectivics of beryllium and rhodium coated mirrors for two angles of incidence. 


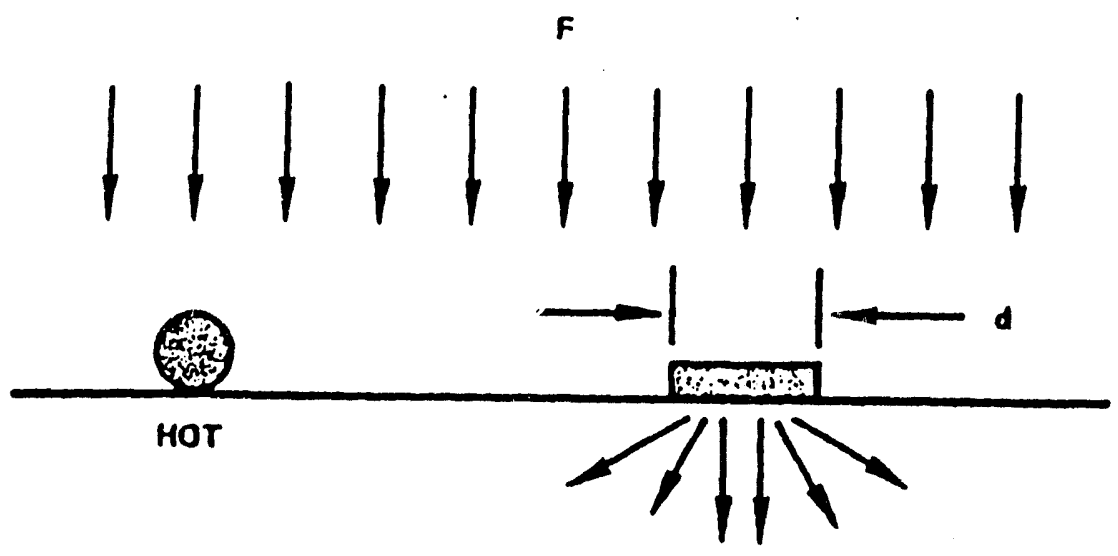

8

Figure 2. Flux $F$ heats defects in poor and good thermal contact with a highly reflecting substrate which has a diffusion depth $\delta$. If defect diameter $d$ is below a critical size, thermal diffusion will limit the temperature of the defect and keep the damage site from growing.

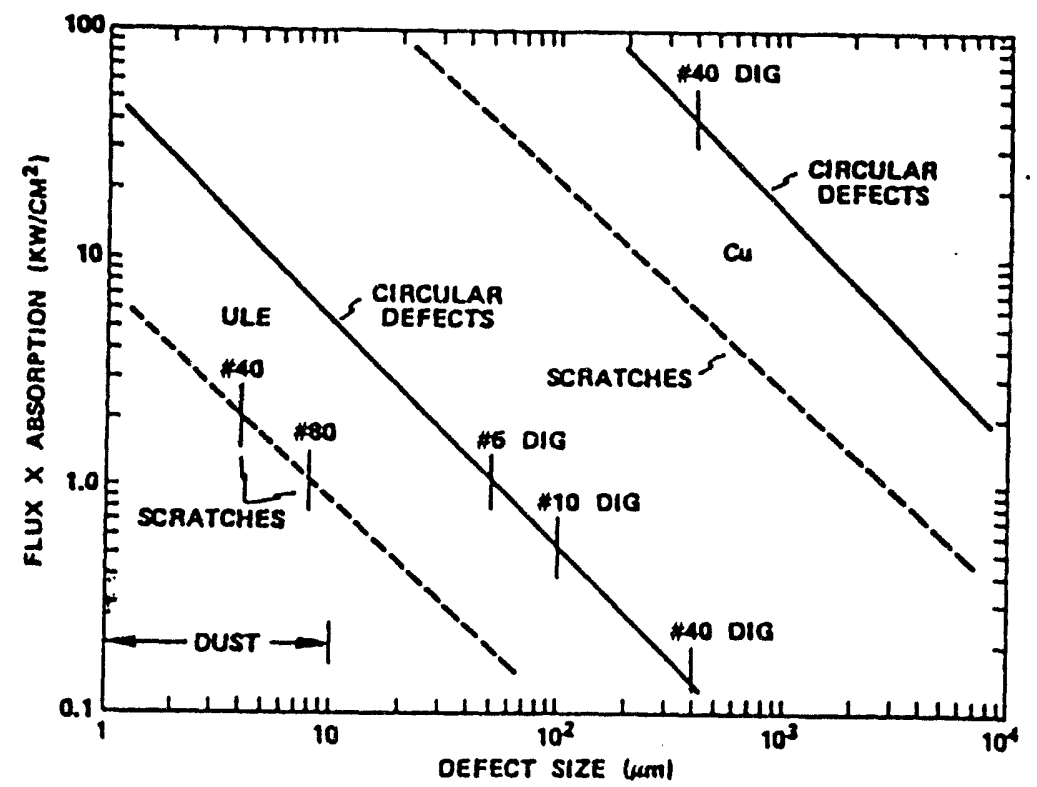

Figure 3. Limiting defect sizes for catastrophic damage under continuous irradiation for ULE quartz substrates (lower left) and copper substrates (upper right). Scratch and dig values are indicated by the vertical lines. 


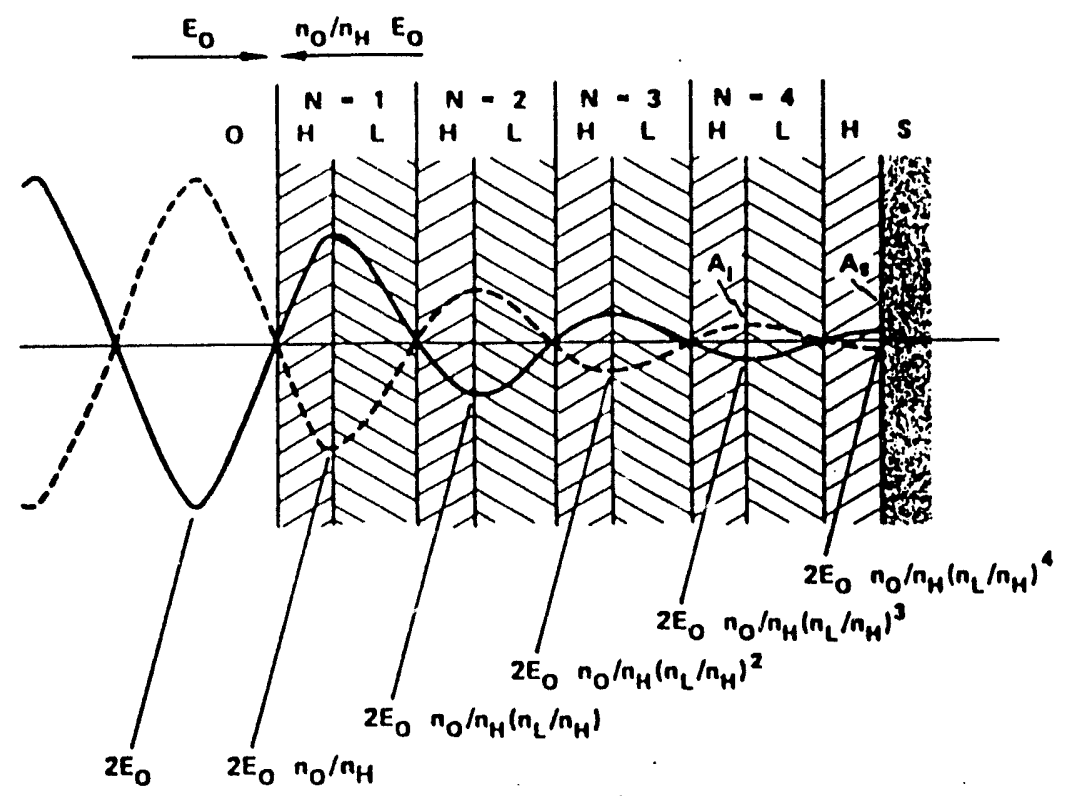

Figure 4. Standing-wave electric fields in a perfect multilayer reflector. At the film-air interface and at each film-pair interface the field becomes negligible. The effect of contaminants on the mirror surface is thus minimized and interface absorption is only important at alternate interfaces. A dielectric substratc is assumed; if it were a metal, the thickness of the initial dielectric layer or layers would be modified, as discussed in the text.

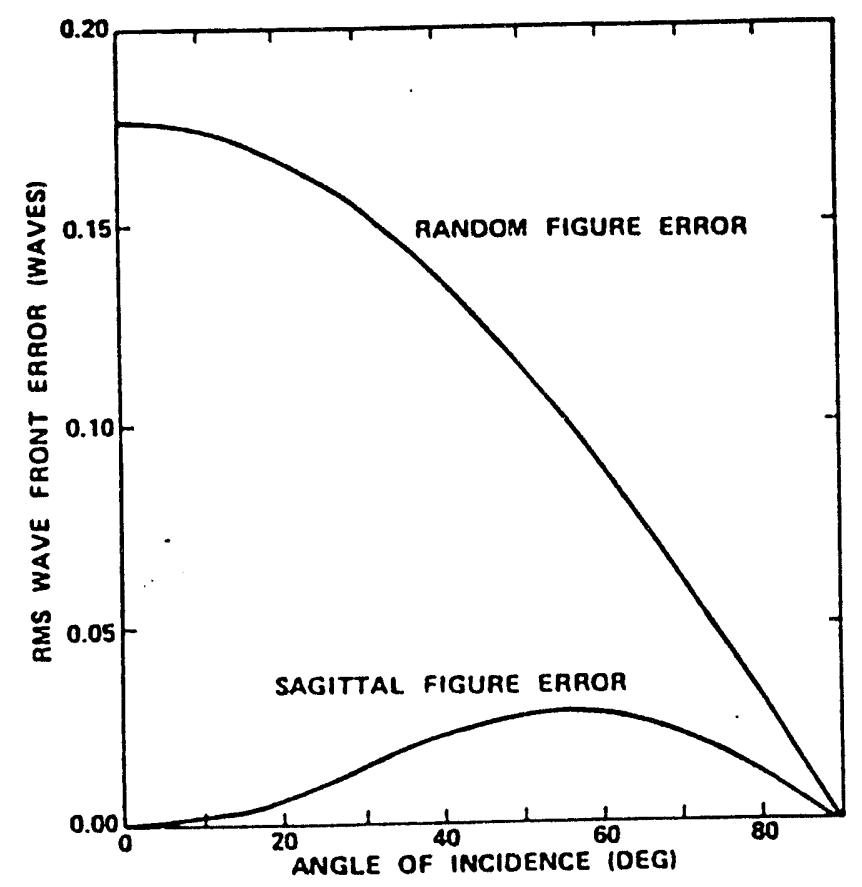

Figure 5. Root-mean-square wave front caused by random peak-to-valley and sagittal quarterwave figure errors. 


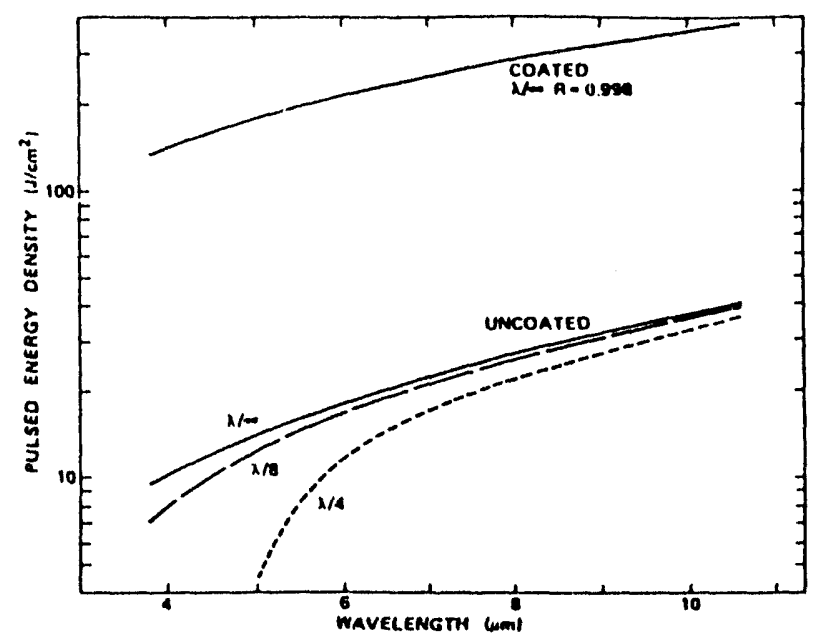

Figure 6. Calculated distortion threshold for polished molybdenum mirrors having various initial figure errors compared to the theoretical performance of a hypothetical molybdenum mirror with no figure error and coated with a 0.998 reflectance coating. Results are for a ten-component system, equal error budget per component, ard a resultant wave front distortion which coss not violate the Mare'chal criterion.

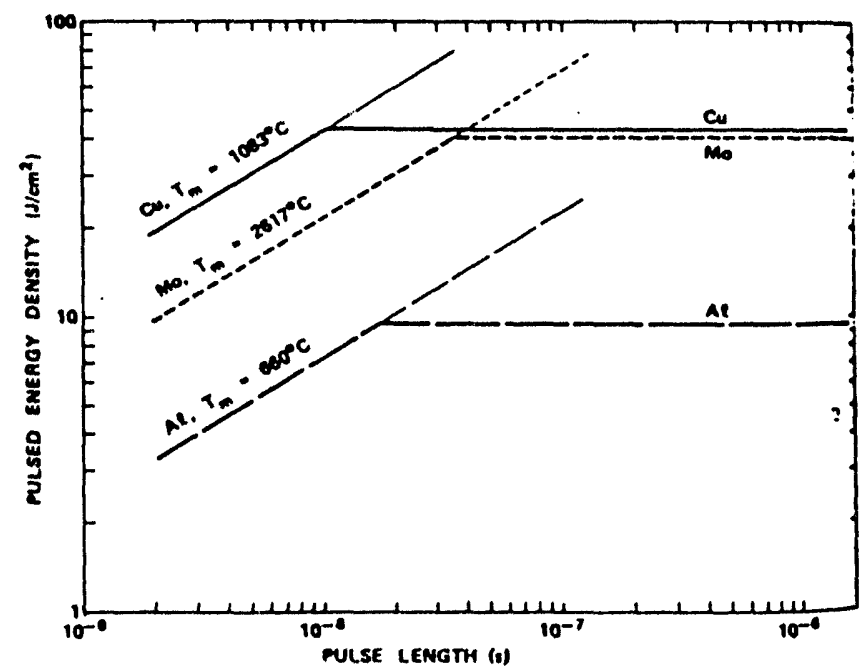

Figure 7. Calculated threshold values of peais energy density for uncoated $\mathrm{Cu}, \mathrm{Mo}$, and $\mathrm{Al}$ mirrors in a ten-component system at a wavelength of $10.6 \mu \mathrm{m}$ as a function of pulse length. Eighth-wave optical figures are assumed. At pulse lengths longer than 10 to 40 ns the threshold value is determined by optical distortion as in previous figures. At shorter pulse lengths it is determined by the catastrophic damage threshold for the mirrors. 
UNCOATED COPPER

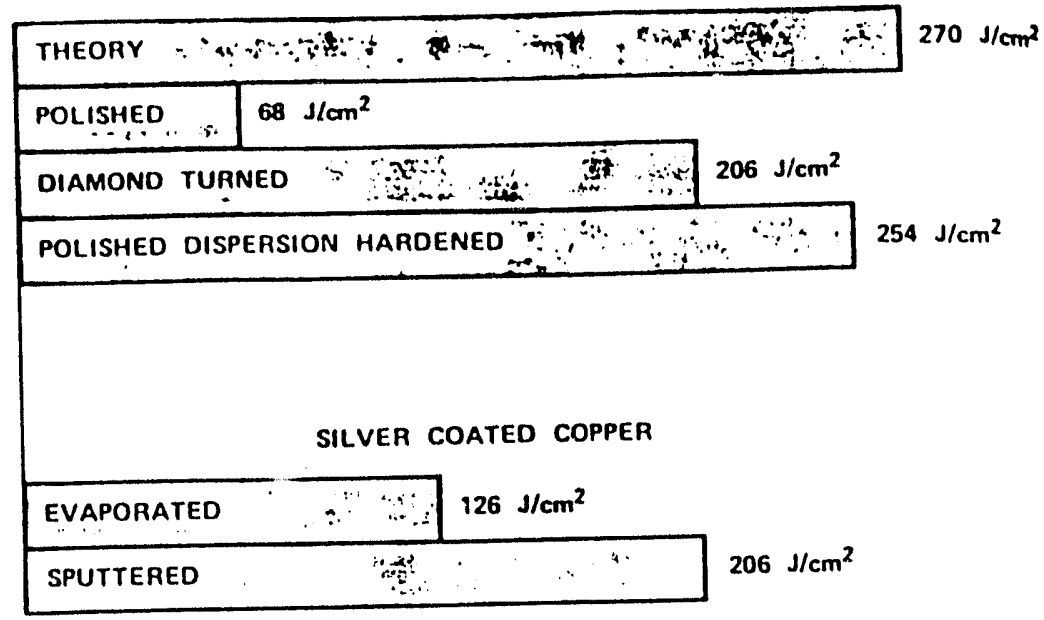

Figure 8. Damage threshold of copper for $10.6 \mu \mathrm{m}$ radiation, $600 \mathrm{~ns}$ pulse length.

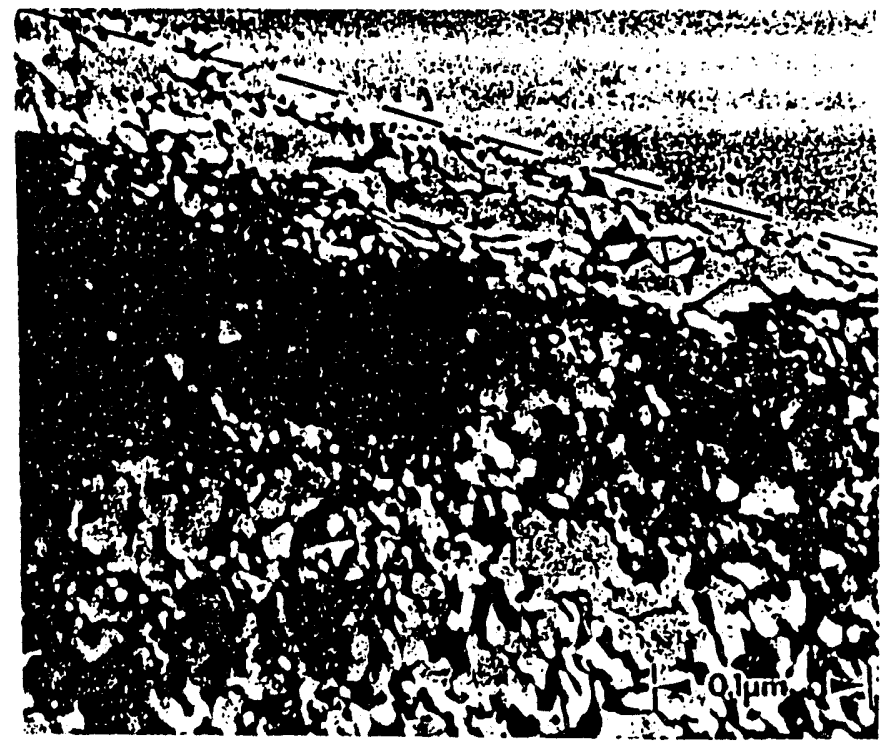

Figure 9. Bright field micrograph of the transverse section of the $-5 \mathrm{deg}$ rake surface. The dashed line is the boundary between the sample (lower) and the $\mathrm{Cu}$ electroplate (above). 


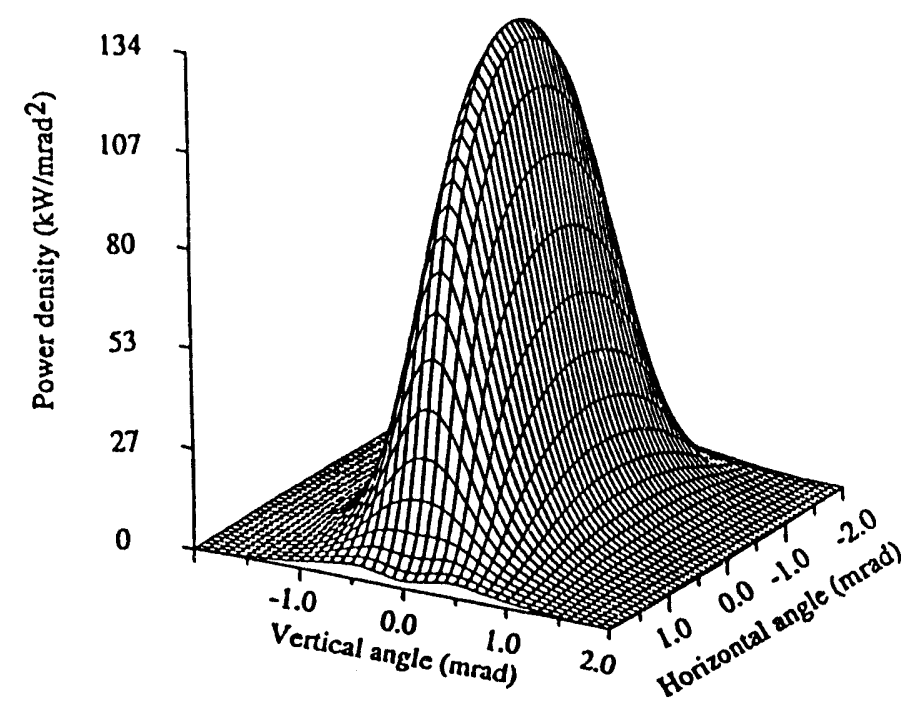

Figure 10. Angular power profile of the undulator beam for the Advanced Photon Source. The peak power density is about $134 \mathrm{~kW} / \mathrm{m} \mathrm{rad}$ and the total power is 3.8 $\mathrm{kW}$.

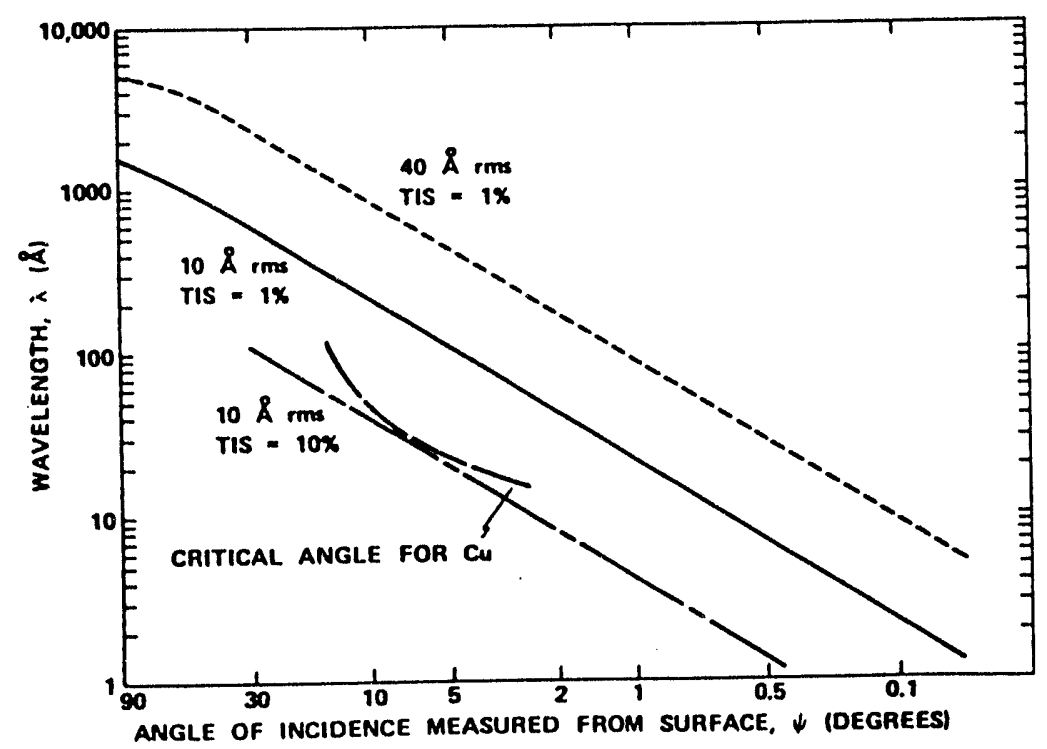

Figure 11. Wavelength dependence of scattered light for different angles of incidence as predicted by Eq. 15. The angle $\psi$ is measured from the surface. The long dashed line shows the experimentally determined critical angle for $\mathrm{Cu}$. 


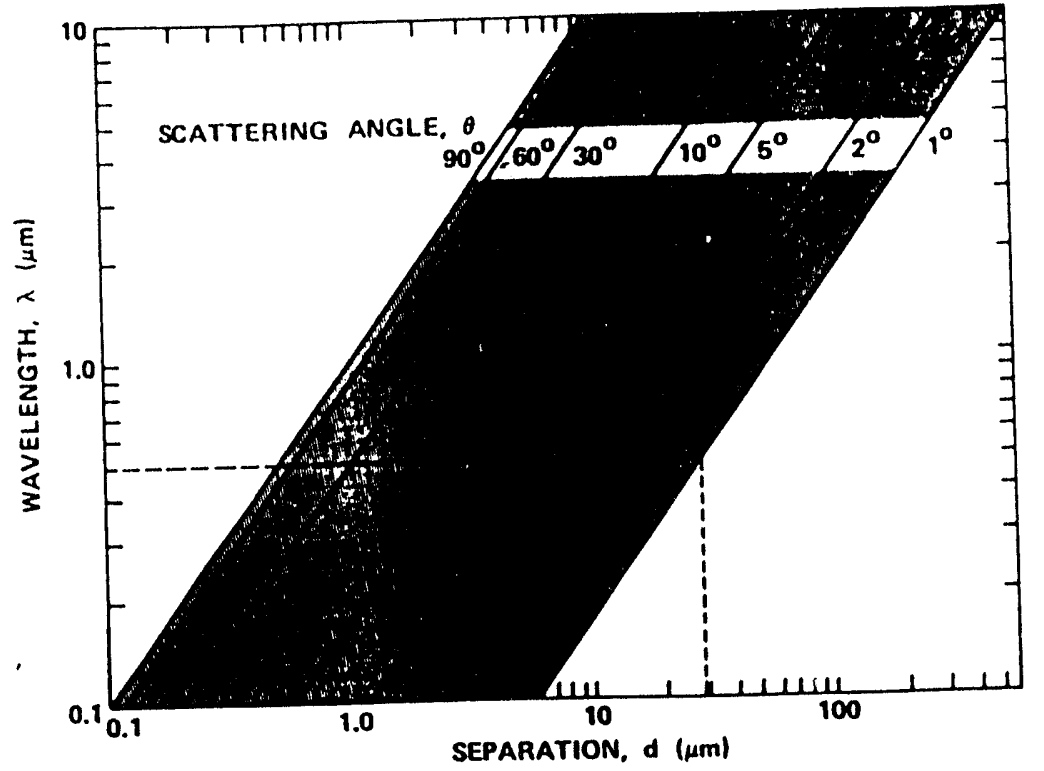

Figure 12. Separations of surface features (spatial wavelengths) which, from the grating equation, would be expected to produce light scattering at angles between 1 and $90^{\circ}$ to the specular beam for normal incidence irradiation. 



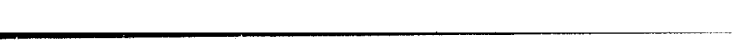

$\longrightarrow$

pof

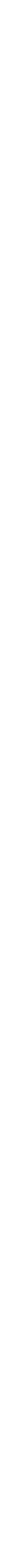

